\title{
Klimaschwankungen im Frühweichsel der Lößabfolgen des Mittelrheingebiets
}

\author{
Wolfgang Boenigk \& Manfred Frechen*) \\ - Loess, palaeosol, lumineszens dating, Greenland ice cores, Tönchesberg, \\ Middle Rhine area, Germany -
}

\begin{abstract}
Zusammenfassung: Am Tönchesberg und in Koblenz-Metternich sind über dem Bt-Horizont des Eems (OIS 5e) bis an die Basis des Oberweichsel-Löß (OIS 2) zehn eigenständige Bodenbildungen nachgewiesen, die durch Sedimentationsphasen voneinander getrennt sind. Bis auf die beiden jüngsten, die als braune Tundrenböden anzusprechen sind, handelt es sich um gut ausgeprägte Ah-Horizonte, z. T. um Bv-, Bt-Ah- bzw. Bt-Horizonte.
\end{abstract}

\section{[Climatic variations in early weichselian loess sequences of the Middle Rhine area]}

Extended Abstract: The loess/palaeosol sequences of the Middle Rhine area provide a relatively detailed and continuous terrestrial record of climate and environment change of the past 200,000 yrs. Loess/palaeosol sequences are generally well pronounced in inter- and intra-crater depressions of scoria cones in the East Eifel Volcanic Field, and on fluvial terraces, e.g. the lower middle terrace of the river Rhine and Moselle. Stratigraphical and chronological investigations of loess/palaeosol sequences from the sections at Tönchesberg and Koblenz-Metternich in the Middle Rhine area, indicate, that the last interglacial/glacial cycle is preserved in much more detail than previously thought. The eruption age of the scoria cones and intercalated tephra layers were independently dated by the $40 \mathrm{Ar} / 39 \mathrm{Ar}$-single grain-fusion dating method. These age estimates are in agreement with the geological interpretation.

In both sections, the last interglacial soil is covered by at least ten palaeosols, postdating the Eemian (oxygen isotope [sub]stage, OIS 5e) brown forest soil. The upper part of the brown forest soil was truncated in both sections. Two A horizons, a chernozem and a brown soil at Tönchesberg and two chernozem-like palaeosols at Koblenz-Metternich, were formed on reworked pedosediments. A reverse magnetization, designated to be the Blake event ( 117,000 yrs), was recognized in these sediments at section Tönchesberg indicating a cooling and accumulation phase between 119,000 and 117,000

\footnotetext{
*) Anschrift der Verfasser: Prof. Dr. W. Boenigk, Abteilung Quartärgeologie, Geologisches Institut, Universität zu Köln, Zülpicher Str. 49a, D-50674 Köln, Priv.Doz. Dr. M. FRECHEN, Centre for Environmental Change \& Quaternary Research, GEMRU, Francis Close Hall, Swindon Road, Cheltenham GL50 4AZ, Großbritannien
}

yrs. It is likely, that this cooling event corresponds to OIS $5 \mathrm{e} 2$. The following warming event, ranging from 117,000 to 113,000 yrs, is evidenced by a chernozem and a brown soil corresponds to OIS $5 \mathrm{e} 1$, as defined by the Greenland ice-core archive. Similar contemporaneous abrupt climate changes were described for the lake sediments of the Lac du Bouchet in France by the pollen spectra during OIS $5 \mathrm{e}$. At the sections of Tönchesberg and Koblenz-Metternich the lower pedocomplex is covered by reworked loess-like sediments and loess, which is designated to be of Lower Weichselian age, most likely OIS $5 \mathrm{~d}$. Mean TL age estimates, although slightly underestimated, (additive dose method) range from 91 to $112 \mathrm{ka}$ for these sediments and are in agreement with the geological interpretation.

The uppermost part of the loess is superimposed by a gleyed chernozem, which is designated to represent the Brörup interstadial (OIS 5c). At section Tönchesberg, the interstadial palaeosol is covered by reworked humic-rich sediments, which are superimposed by a "parabraunerde-chernozem (BtAh)" palaeosol, designated to represent the Odderade interstadial and OIS $5 \mathrm{a}$. Pellet sands and reworked humic-rich sediments intercalated by at least three soil formations (A horizons) were accumulated during OIS 4 and 3 . It is likely that these soil formations are younger than 75,000 and older than 50,000 yrs based on luminescence dating.

A correlation between the terrestrial record of the loess/palaeosol sequences from the Middle Rhine area and the deep sea climate archive is difficult owing to the low resolution and to the climatic response of the oxygen isotope ratio, which primarily records the continental ice volume. As climate changes over western Europe and Greenland might have been contemporaneous throughout the past 130,000 yrs, a correlation with the ice-core record is likely. The loess/palaeosol sequences in the Middle Rhine area show a similar succession in time and a similar number of climatic fluctuations for the Lower Weichselian (OIS 5), as recorded in the ice core archive. Thus, cooling and warming events, recorded in the GRIP ice core, may have been strong enough to cause a marked change in the vegetation and hence in the surface stability of Central Europe. It is still an open question whether these fluctuations are synchronous or out of phase.

During late OIS 5e up to early OIS 3 in Central Europe, the climate and environment change indicates a corre- 
lation with variation of temperature during times of no or little ice in North America and Eurasia. A more reliable correlation between the Central European loess archive and the Greenland ice-core record improves the climate reconstruction of the past 130,000 yrs.

\section{Einleitung}

Gut gegliederte Weichsellöß-Abfolgen werden von BrunNaCker (1958) und Semmel (1968) aus dem Mittelrhein-, dem Rhein-Main-Gebiet und aus Süddeutschland beschrieben. Die aus diesen Untersuchungen resultierenden Standardabfolgen des letzten Interglazial-/Glazialzyklus beginnen mit einer Parabraunerde, die mit dem Klimaoptimum der letzten Warmzeit, dem Eem-Interglazial, korreliert wird. BRuNNACKER (1990) unter- gliedert das Frühweichsel neben humosen Fließerden in eine Untere und eine Obere Humuszone. BRUNNACKER (1958) beschreibt aus Kitzingen in Süddeutschland drei Humuszonen oberhalb des letztinterglazialen Bodens. SEMmeL (1968) differenziert im Rhein-Main-Gebiet drei Humuszonen, Mosbacher Humuszonen, die sich oberhalb des „Erbacher Bodens" (Äquivalent des Eems), während der frühweichselzeitlichen Interstadiale bildeten.

In den letzten 10 Jahren erbrachten lößstratigraphische Untersuchungen im Mittelrheingebiet eine kompliziertere Abfolge und detailliertere Gliederung der frühweichselzeitlichen Löß-Paläoboden-Abfolgen. Die derzeit am besten gegliederten frühweichselzeitlichen Lößsequenzen sind an der

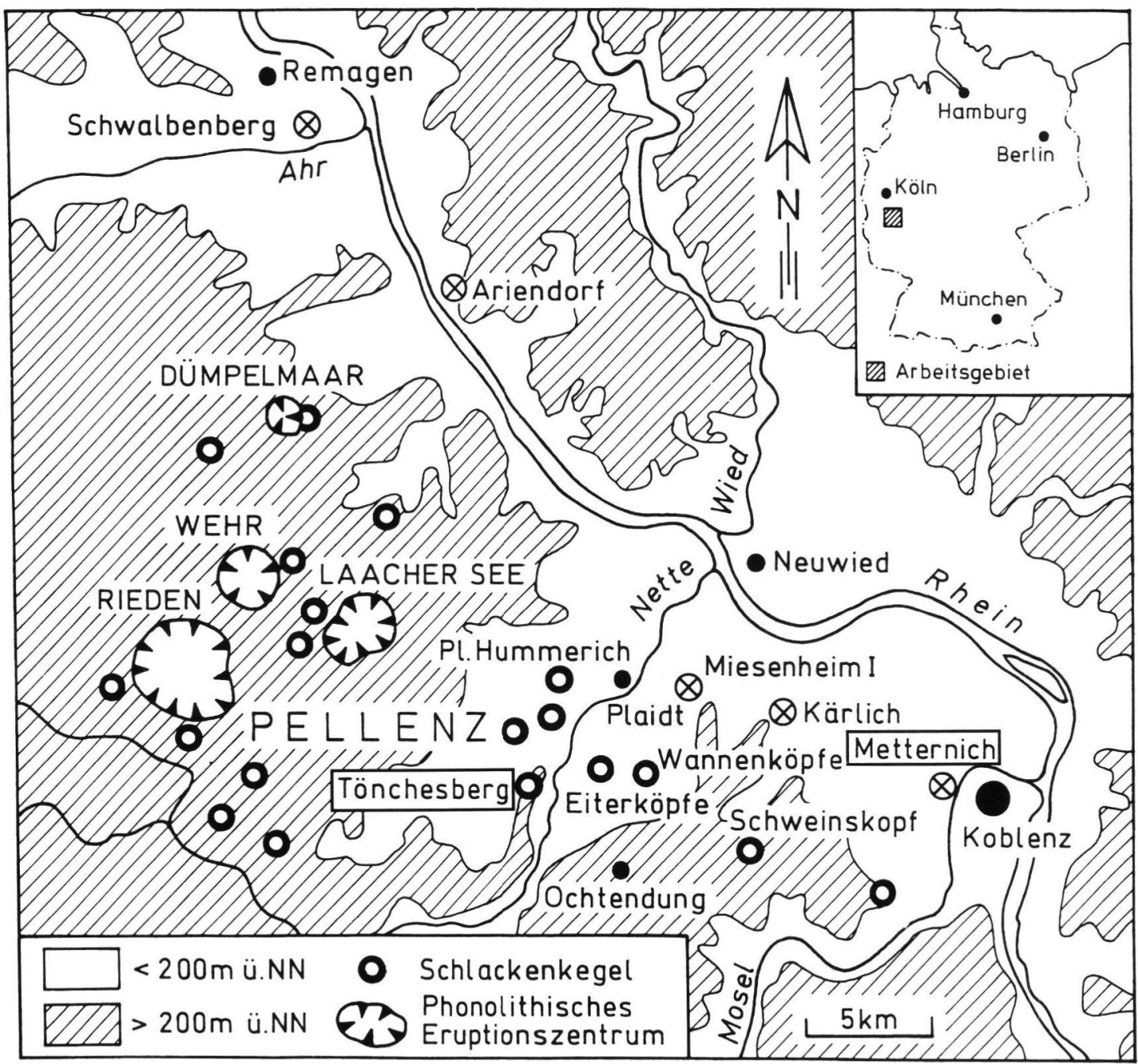

Abb. 1: Lage der Profile Tönchesberg und Koblenz-Metternich am Mittelrhein

Fig. 1: Map showing the locality of the Tönchesberg and Koblenz-Metternich section in the lower Middle Rhine area 
Mosel in Koblenz-Metternich (s. Abb. 1) (BoENIGK et al., 1994, 1999; FreCHEN et al., 1995; CONARD et al. 1995) und in der Osteifel am Tönchesberg (Becker et al., 1989; Hentzsch, 1990; Zöller et al. 1989; BoENIGK \& FreCHEN, 1994; FreChen, 1994; Reinders \& Hambach, 1995; Boenigk et al., dieser Band) sowie an den Wannenköpfen (FreCHEN, 1995; FreCHEN \& Justus, 1998) aufgeschlossen. Im folgenden sollen die Abfolgen vom Tönchesberg und von Koblenz-Metternich einander gegenübergestellt werden.

Tönchesberg:

Am Tönchesberg, einem Schlackenvulkan aus dem jüngeren Mittelpleistozän, sind saale- und weichselzeitliche Löß-Deckschichten in großer Mächtigkeit erschlossen. In den Deckschichten des Tönchesberges wird der unterste zwischengeschaltete rotbraune Bt-Horizont einer $\mathrm{Pa}$ rabraunerde mit dem Klimaoptimum der letzten Warmzeit, dem Eem, korreliert. Diese Interpretation basiert auf der vulkanologischen Altersabschätzung des Tönchesberg-Vulkans, die eine Einschätzung jünger als die Hüttenberg-Tephra mit $215 \pm 4$ ka erlaubt (BOGAARD \& SCHMINCKE 1990), so daß nur saalezeitliche und jüngere Deckschichten möglich sind. Der Nachweis des Blake-Event (BECKER et al. 1989, REINDERS \& HAMBACH 1995) der magnetischen Polaritäts-Skala mit 117 ka oberhalb des 1. kräftigen Bt-Horizontes in der Deckschichtenfolge bestärkt die pedologische Einstufung dieses Bt-Horizontes als Rest der Parabraunerde des Eem-Interglazials (Unterstufe 5e). Damit sind die hangenden Ablagerungen sicher eem- bis weichselzeitlichen Alters, was durch die Lumineszenz-Daten (FrECHEN 1994) bestätigt wird.

Diese weichselzeitlichen Ablagerungen zeigen ein reich gegliedertes Profil, das 11 Bodenhorizonte aufweist (Boenigk et al. dieser Band), von denen 10 Horizonte in das Unter- und MittelWeichsel zu stellen sind (Abb. 2). Diese Bodenhorizonte wurden durch Geländeansprache, Messung der toc- (total organic carbon), Karbonatund Ton-Gehalte sowie durch mikromorphologische Untersuchungen nachgewiesen. Es ergibt sich folgende Sequenz für das Unter- und Mittelweichsel

\section{Löß: Oberweichsel}

10 brauner Boden

Fließserde

9 brauner Boden
Fließerde

Diskordanz

8 Ah-Horizont

Fließerde, humos

7 Ah-Horizont

Lehmbröckel-Sande

Diskordanz-Erosion

Löß, vom Hangenden Frostspalten

6 Ah-Horizont, Schwammgefüge

Fließerde, humos, Blöcke an der Basis

Diskordanz-Erosion

5 BtAh-Horizont Parabraunerde-Tschernosem: Schwammgefüge, Tonverlagerung, Ton und

Humusverarmung zum Hangenden

Lehmbröckelsand u. Fließerde

Diskordanz

Fließerde, humos

Diskordanz-Erosion

4 Ah-Horizont Tschernosem eines feuchten

Standorts (SwAh), im tieferen Teil

synsedimentäre Überprägung

Schwemmlöß- und Fließerde

Diskordanz-Erosion

3 Ah-Horizont, Tschernosem, intensive Bioturbation, makroskopisch gut sichtbar am Übergang vom Löß in den Ah-Horizont Schwemmlöß

Diskordanz

2 Bv-Horizont: stark humos, dünne Tonbeläge

1 Ah-Horizont stark humos, Bioturbationsspuren Fließerde, humos mit Blake Event 117ka

Diskordanz

Bt-Horizont $=$ Eem $($ Unterstufe 5 e $)$

Koblenz-Metternich:

Die Deckschichten der Unteren Mittelterrasse der Mosel in Koblenz-Metternich beginnen mit Hochflutsedimenten und Löß, der zu einem Bt-Horizont einer Parabraunerde überprägt wurde. Dieser interglaziale Boden wird aufgrund der Terrassenstratigraphie (Untere Mittelterrasse $=$ saalezeitliche Ablagerung) und der Lumineszenz-Datierungen an den Deckschichten mit dem Klimaoptimum des letzten Interglazials, der EemWarmzeit, korreliert (BoENIGK et al., 1994; FRECHEN et al., 1995). Darüber folgen diskordant humose Fließerden, in denen zwei Humuszonen, Ah-Horizonte, zwischengeschaltet sind. Diese Abfolge wird von BoEnIGK et al. (1999) als unterer Pedokomplex bezeichnet. Darüber sind Lehmbröckelsande und Schwemmlösse abgelagert worden. Zum Hangenden hin folgt der obere Pedokomplex, der aus zwei kräftigen Bt-Horizonten, die durch einen Ah-Horizont (Tschernosem) diskordant voneinander getrennt sind, aufgebaut wird. 
Tabelle 1: Ergebnisse der Paläodosis- und Dosisleistungsbestimmung sowie IRSL-Alter. Die Probenbezeichnungen sind identisch mit denen aus FRECHEN (1994).

Table 1: Results of the palaeodose and dose rate determination and IRSL age estimates. The sample numbers are identical with those described in FRECHEN (1994).

\begin{tabular}{|l|l|l|l|l|}
\hline Probe & $\begin{array}{l}\text { Palaeodosis } \\
{[\text { Gray }]}\end{array}$ & $\begin{array}{l}\text { Dosisleistung } \\
{[\text { Gy/ka] }}\end{array}$ & $\begin{array}{l}\text { IRSL-Alter } \\
{[1000 \text { Jahre }]}\end{array}$ & $\begin{array}{l}\text { Mittelwerte } \\
\text { in Abb. }\end{array}$ \\
\hline Toe 9 & $51,3 \pm 3,5$ & 3,821 & $13,4 \pm 1,3$ & 13 \\
\hline $\begin{array}{l}\text { Toe } 12 \\
\text { Toe } 13\end{array}$ & $96,0 \pm 5,7$ & 3,582 & $26,8 \pm 1,8$ & $25-27$ \\
\hline Toe 20 & $104,9 \pm 4,6$ & 4,179 & $25,1 \pm 2,3$ & \\
Toe 22 & $156,8 \pm 11,4$ & 4,031 & $38,9 \pm 4,6$ & $39-40$ \\
\hline Toe 24 & $161,3 \pm 7,5$ & 4,053 & $39,8 \pm 3,9$ & 48 \\
\hline Toe 50 & $174,2 \pm 6,6$ & 3,607 & $48,3 \pm 4,2$ & 64 \\
\hline Toe 40 & $226,1 \pm 14,0$ & 3,510 & $64,4 \pm 6,6$ & 75 \\
\hline
\end{tabular}

Darüber wurden humose Fließerden und Lehmbröckelsande akkumuliert, die ihrerseits mindestens durch drei Ah-Horizonte untergliedert werden. Die humosen Fließerden werden von Schwemmlössen und lößartigen Fließerden überlagert, die durch 3 Diskordanzen unterteilt werden. Sie sind durch markante Kiesschnüre gekennzeichnet.

Aufgrund der detaillierten Lumineszenz-Datierungen (Tab. 1) ist die Grenze zu den OberWeichsel-Ablagerungen an der 1 . oder 2 . Kiesschnur festzulegen (Abb. 2).

\section{Korrelation:}

Mit den beiden Schichtenfolgen vom Tönchesberg und von Koblenz-Metternich (Abb. 2) liegen Akkumulations/Bodenbildungssequenzen für das Unter- und Mittel-Weichsel vor, die über dem Bt-Horizont des Eem-Interglazials, am Tönchesberg 10 und in Koblenz-Metternich 8 Bodenhorizonte aufweisen. In Koblenz-Metternich kommen noch 2 Diskordanzen hinzu.

Die Postulierung einer solchen Vielzahl von Böden im Zeitabschnitt Unter- und Mittel-Weichsel muß zu Widerspruch herausfordern, daher hier die Diskussion einiger möglicher Einwände.

1. Einwand: Es handelt sich um eine Ausnahme.

Jedes gute Profil mit einer Detail-Gliederung ist ein Ausnahmefall. Nur die wenigsten Ereignisse des letzten Glazials sind in Löß-Profilen kontinuierlich überliefert.

\section{Einwand: Stimmt der Zeitrahmen?}

Für den Tönchesberg ist durch vulkanologische Bearbeitung, gestützt auf radioisotopische Datierung in Kombination mit Paläomagnetik, Lumineszenzdatierung und nicht zuletzt sedimentologisch-pedologische Bearbeitung die Altersstellung eindeutig.

Das Profil Koblenz-Metternich liegt auf der Unteren Mittelterrasse, die bislang unwidersprochen in das Saale-Glazial i.e.S. gestellt wird. Die Deckschichten sollten dann in das Eem-Interglazial und in die Weichsel-Kaltzeit zu stellen sein. Hier könnte eine Fehleinstufung vorliegen, doch paläomagnetische Untersuchungen und Lumineszenzdatierungen (Abb. 2) an den Deckschichten bestätigen die Einstufung dieser Ablagerungen in das EemInterglazial und das Weichsel-Glazial.

3. Einwand: Die beschriebenen Phänomene werden überbewertet.

Dem ist entgegenzuhalten, daß es nicht zu bestreiten ist, daß in beiden Profilen eine Wechselfolge von Akkumulation und Bodenbildung vorliegt.

Die Bodenbildungen sind durch Geländeansprache, toc-, Karbonat- und Tonmineral-Gehalt sowie durch Mikromorphologie (BOENIGK et al. 1999; Boenigk et al. 2000) gut belegt. Es bleibt das Argument, es handele sich um sehr kurze Zeitabschnitte, und der klimatische 


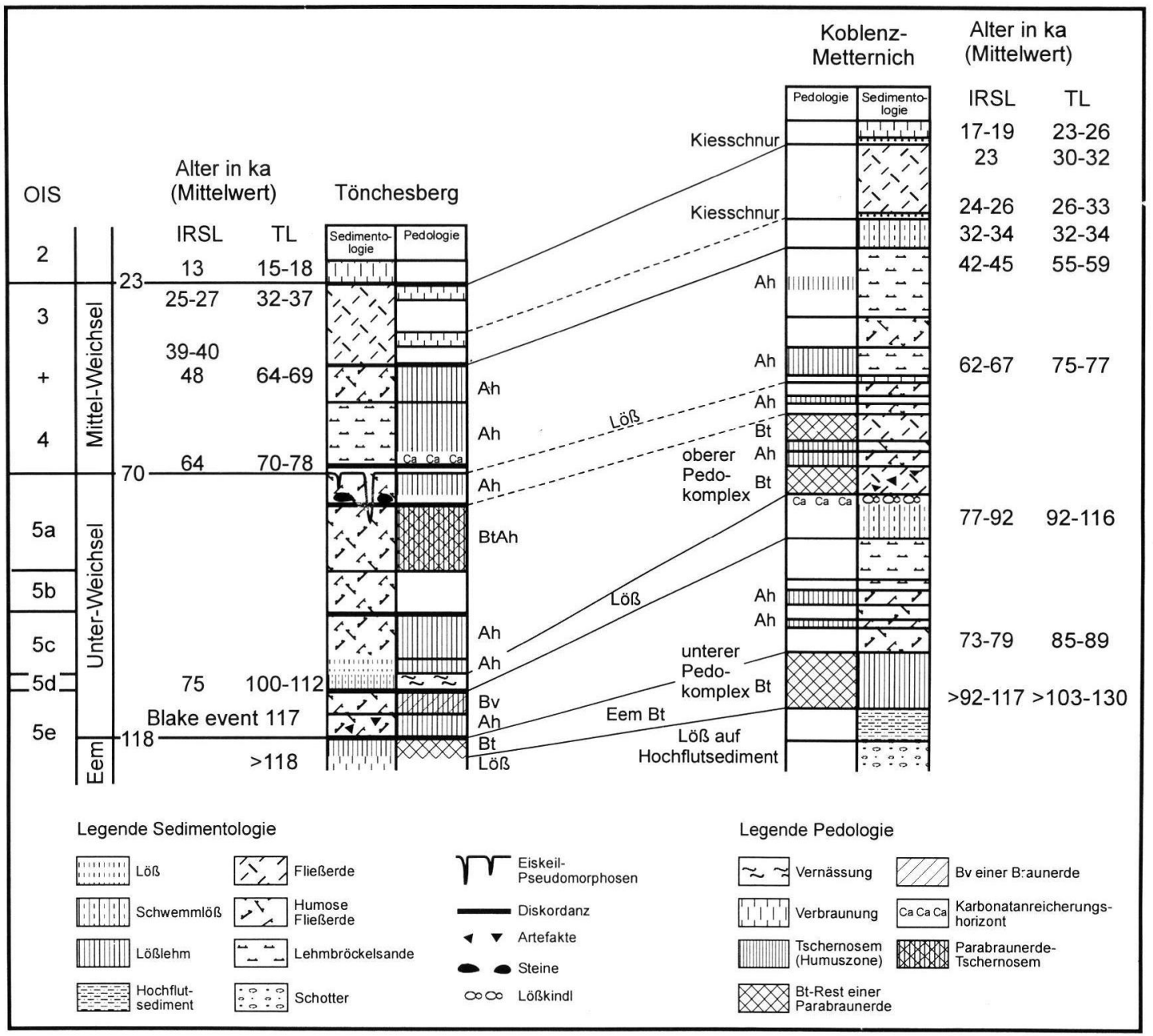

Abb. 2: Korrelation der Löß-/Paläoboden-Abfolgen des Tönchesberges und von Koblenz-Metternich. Die TL-Datierungsergebnisse Tönchesberg sowie die IRSL- und TL-Datierungsergebnisse von Koblenz-Metternich sind aus FrECHEN (1994) und FrECHEN et al. (1995) entnommen. Die neuen IRSL-Datierungsergebnisse Tönchesberg beziehen sich auf Tabelle 1.

Fig. 2: Correlation of the loess/palaeosol sequence from the sections at Tönchesberg and Koblenz-Metternich $(\mathrm{Bt}=$ clay-rich $\mathrm{B}$ horizon of brown forest soil; $\mathrm{Ah}=$ humic-rich A horizon). TL age estimates concerning Tönchesberg and IRSL and TL age estimates concerning Koblenz-Metternich are from FRECHEN (1994) and FRECHEN et al. (1995). The data of the IRSL age estimates for Tönchesberg are found in table 1.

Wechsel sei unerheblich. Sicher ist, daß es sich bei den meisten der beschriebenen Bodenbildungen um sehr kurze Phasen handelt, aber ihre Dauer und klimatische Ursache ist mit Sicherheit höher zu bewerten als die der Naßböden im Oberweichsel, die als stratigraphische Leithorizonte verwendet werden.

Die Frage der Wertigkeit der Sedimentationsergebnisse zwischen den Phasen der Bodenbildung bleibt offen. In den meisten Fällen handelt es sich um Fließerden und verspülte Sedimente, in weni- gen Horizonten um Löß, der kaltzeitliche Bedingungen anzeigt. Fließerden und Verspülungshorizonte deuten aber ebenfalls auf eine instabile Landoberfläche, die klimatisch und durch geringe Vegetation zu erklären ist.

Die Korrelation der beiden Profile ergibt folgende Ergebnisse (Abb. 2): Die Verknüpfung der Bt-Horizonte, die als Relikte der Parabraunerde aus dem Eem (5e) aufgefaßt werden, ist nach dem vorliegenden Daten-Material anzunehmen. Ebenso klar lassen sich die Lösse des Ober-Weichsels 
aufgrund der Lumineszenz-Datierungen identifizieren (FreCHEN 1994; FrECHEN et al. 1995)

Das zwischen diesen beiden Einheiten liegende Sedimentpaket muß daher in das Unter- und Mittel-Weichsel gestellt werden. Für diesen Zeitabschnitt liegen als Datierungshilfen am Tönchesberg die Identifizierung des Blake-Event (BECKER et al. 1989, Reinders \& Hambach 1995) und für beide Profile Lumineszenz-Datierungen vor (FreCHEN 1994, FRECHEN et al. 1995). Die Ablagerungen - Fließerden und verspülte Sedimente - sind jedoch für Thermolumineszenz-Datierungen nicht ideal, so daß noch Unsicherheiten bestehen. Die Korrelation (Abb. 2) erfolgt daher im wesentlichen aufgrund sedimentologisch/pedologischer Ansprache unter Berücksichtigung der Infrarot Optisch Stimulierten Lumineszenz-Daten.

Der untere Pedokomplex von Metternich wird mit dem Eem-Bt und den darüber folgenden Artefakte-führenden Ablagerungen mit den Bodenhorizonten eines Ah- und eines Bv-Horizontes am Tönchesberg verknüpft.

In beiden Profilen folgt darüber ein mehrere Meter mächtiges Lößpaket.

Der obere Pedokomplex von Metternich mit seinen beiden Bt-Horizonten wird mit den sehr kräftigen Bodenhorizonten von Tönchesberg, die bei BECKer et al. (1989) als erste und zweite UnterWeichsel-Humuszone bezeichnet werden, korreliert.

Aufgrund der Lumineszenz-Datierungen wird im Top des Bt-Ah-Horizontes am Tönchesberg und im Top des obersten Bt-Horizontes in KoblenzMetternich die Grenze zwischen Unter- und Mittel-Weichsel angenommen.

Auf eine weiterführende Parallelisierung wird verzichtet, da angenommen werden muß, daß auch diese detaillierten Abfolgen nicht vollständig sind und eine Verknüpfung nur nach der $\mathrm{Ab}$ zähl-Methode fragwürdig ist. Gewisse Lnterschiede in der Ausprägung der Böden sind sicher standortbedingt. Koblenz-Metternich liegt im geschützten Moseltal, der Tönchesberg dagegen 14 km entfernt auf der Hochfläche der Eifel und morphologisch ca. $150 \mathrm{~m}$ höher.

\section{Diskussion und Schlußfolgerungen}

Zusammenfassend sind sowohl am Tönchesberg als auch in Koblenz-Metternich zwischen dem BtHorizont des Eems (Sauerstoff-Isotopenstadium 5e) und der Basis des Oberweichsel-Lösses (Sauerstoff-Isotopenstadium 2) 10 eigenständige Bodenbildungen nachgewiesen, die durch Sedimen- tationsphasen voneinander getrennt sind. Bis auf die beiden jüngsten, die als braune Böden anzusprechen sind, handelt es sich um gut ausgeprägte Bodenhorizonte, die als Ah-Horizonte, die z.T. zu einem Bv-, BtAh- bzw. Bt-Horizont entwickelt sind.

Eine Korrelation mit dem marinen Klima-Archiv erweist sich für das Frühweichsel als schwierig bzw. nicht durchführbar. Die Auflösung der marinen Sauerstoff-Isotopenkurve reicht nicht aus (MARTINSOn et al. 1987; Bassinot et al. 1994), um die zahlreichen Paläoböden zu korrelieren.

Die Korrelation des untersten kräftigen Bt-Horizontes am Tönchesberg mit dem Sauerstoff-Isotopen-Substadium 5e kann aufgrund der Chronologie und der stratigraphischen Ergebnisse als gesichert angesehen werden. Der unterste Bt-Horizont der Parabraunerde von Koblenz-Metternich wird aufgrund der Lumineszenz-Datierungen (Frechen et al. 1995) und dem Vorhandensein der Deckschichten auf der saalezeitlichen Unteren Mittelterrasse mit dem Klimaoptimum der letzten Warmzeit korreliert.

Am Tönchesberg werden die beiden im Hangenden folgenden Ah-Horizonte, der oberste zu einer Braunerde degradiert, aufgrund der inversen Magnetisierung, die mit dem Blake Event korreliert wird (Becker et al., 1989; Reinders \& Hambach, 1995) und aufgrund der Lumineszenz-Datierungen (FrECHEN 1994) zeitlich in das ausgehende Sauerstoff-Isotopen-Substadium 5 e gestellt.

Der kräftige Parabraunerde-Tschernosem am Tönchesberg wird aufgrund der LumineszenzChronologie in das Sauerstoff-Isotopenstadium 5 a gestellt. Der oberste Bt-Horizont des oberen Pedokomplexes aus Koblenz-Metternich wird mit dieser kräftigen Bodenentwicklung korreliert.

Die darüber folgenden drei schwachen Ah-Horizonte sowie die beiden schwachen Verbraunungen, die mit den beiden Lohner Böden korreliert werden, sind in beiden Profilen aufgrund der Lumineszenz-Datierungen in das Mittel-Weichsel, vermutlich in das frühe Stadium $3 \mathrm{zu}$ stellen.

Ein Vergleich dieser früh- und mittelweichselzeitlichen Löß-/Paläobodenabfolgen mit den palynologisch untersuchten Weichsel-Interstadialen in Niedersachsen (BeHRE 1989; BeHRE \& LADE 1986; Behre \& VAN DER Pflicht 1992) legt nahe, daß aufgrund der chronologischen Daten der Tschernosem-Pseudogley mit dem Brørup und der kräftige Parabraunerde-Tschernosem mit dem Odderade korreliert werden. Die darüber folgenden schwächer ausgebildeten Ah-Horizonte lassen 
sich mit dem Oerel-, Glinde-, Moershoofd- und Hengelo-Interstadial korrelieren. Der obere schwachbraune Boden der oberen Mittelweichselzeitlichen Abfolge des Tönchesberges kann mit dem Denekamp-Interstadial korreliert werden.

Die Lößprofile lassen aber darüber hinaus, zwischen dem Bt-Horizont des Eem und der Bodenbildung, die dem Brørup gleichgesetzt wird, mehrere Klimaschwankungen erkennen.

Die Abfolge über dem Bt vom Tönchesberg beginnend mit einer Diskordanz gefolgt von der Ablagerung einer Fließerde mit dem paläomagnetischen Signal des Blake-Event und den Bodenhorizonten 1 und 2, muß noch in Sauerstoff-Isotopen-Unterstufe 5 e eingeordnet werden und belegt Klimaschwankungen im ausgehenden Eem.

Über dem folgenden Lößpaket, das dem Sauerstoff-Isotopen-Stadium $5 \mathrm{~d}$ zuzuordnen ist, erfolgt eine Bodenbildung aus Löß, danach eine Erosion, dann die Akkumulation und Bodenbildung, die dem Brørup zugeordnet werden kann; das heißt, vor dem eigentlichen Brørup hat es eine weitere Klimaschwankung gegeben, die wie das Brørup in Sauerstoff-Isotopen-Unterstufe $5 \mathrm{c}$ einzustufen ist.

Es wird angenommen, daß die Klima- und Umweltveränderungen während der letzten 130.000 Jahre im westlichen Europa und in Grönland isochron abgelaufen sind (DANSGAARD et al. 1993). Daher liegt ein Vergleich der sehr detaillierten Löß-/Paläoboden-Abfolge des „Eiszeitlichen Lößprofils“ und vom Tönchesberg, Osteifel, mit den Klimadaten des GRIP-Eisbohrkernes für das Frühund Mittelweichsel nahe. Neben den signifikanten Klimaveränderungen, z.B. das letzte Interglazial und die beiden frühglazialen Interstadiale Brørup und Odderade, wurden mehrere Klimaschwankungen mit geringerer Intensität nachgewiesen. Diese Klimaveränderungen waren genügend ausgeprägt, um die Vegetation und damit die Oberflächenstabilität in Mitteleuropa zu beeinflussen und konnten so in den beiden LößProfilen überliefert werden. Ein Vergleich mit den Daten aus den Eisbohrkernen, z. B. bei JoHnson et al. 1997, zeigt große Ähnlichkeit der Zahl und Position der Klimaschwankungen, was als isochroner Verlauf der Klimakurve von Mitteleuropa und von Grönland interpretiert werden kann.

Die vorgelegten stratigraphischen und chronologischen Daten zeigen, daß der letzte Interglazial/Glazialzyklus in den Lößsequenzen Mitteleuropas wesentlich besser überliefert ist als bisher ver- mutet. Im Vergleich mit den Klima-Archiven aus Eisbohrkernen, See-Sedimenten und Mooren zeigt sich, daß in Lößprofilen eine durchaus gleichwertige Differenzierung des Klimaganges möglich ist.

\section{Dank}

Wir bedanken uns bei der DFG für die finanzielle Förderung im Rahmen des Projektes „Quartär der Eifel" (Bo 413/5). Frau Krings sorgte für die hohe Qualität bei den Reinzeichnungen der Abbildung.

\section{Schriftenverzeichnis}

Bassinot, F. C., Labeyrie, L. D., Vincent, E., Quidelleur, X., Shackleton, N.Y. \& Lancelot, Y. (1994): The astronomical theory of climate and age of the Brunhes-Matuyama magnetic reversal.- Earth and Planetary Science letters, 126: 91-108; Elsevier.

Becker, U., Boenigk, W. \& Hentzsch, B. (1989): Reverse Magnetisierung in den frühwürmzeitlichen Deckschichten am Tönchesberg/Osteifel.- Mainzer Naturwissenschaftliches Archiv, 27: 1-22; Mainz.

BEHRE, K.-E. (1989) Biostratigraphy of the last glacial period in Europe.- Quaternary Science Reviews, 8: 25-44;

- \& Lade, U. (1986): Eine Folge von Eem und 4 Weichsel-Interstadialen in Oerel/Niedersachsen und ihr Vegetationsablauf.- Eiszeitalter u. Gegenwart, 36: 11-36; Hannover.

— \& Pflicht, J. v.d. (1992): Towards an absolute chronology for the last glacial period in Europe: radicarbon dates from Oerel, northern Germany.- Vegetation History and Archaeobotany, 1: 111-117;

BOENIGK, W. \& Frechen, M. (1994): Mittel- und oberpleistozäne Deckschichten des Tönchesberges, Osteifel.- In: SchIRMER, W. (Hrsg.): Landschaftsgeschichte im europäischen Rheinland. - Rheinlandverlag; Köln (im Druck).

- \& Weidenfeller, M. (1994): Die mittel- und oberpleistozäne Deckschichtenfolge im Naturschutzgebiet „Eiszeitliches Lößprofil“ in Koblenz-Metternich.Mainzer Geowissenschaftliche Mitteilungen, 23, S. 287-320, 14 Abb., Mainz.

- \& Schweitzer, U. (1999): Mikromorphologische Charakterisierung der Deckschichten von Kärlich und Koblenz-Metternich. - Mainzer geowissenschaftliche Mitteilungen, Mainz (im Druck).

- \& SCHWEITzer (2000): Mikromorphologische Charakterisierung der Deckschichten des Tönchesberges. - Eiszeitalter und Gegenwart; Krefeld.(zum Druck eingereicht)

BogaARd, P. v. d. \& SChmincke, H.-U. (1990) Die Entwicklungsgeschichte des Mittelrheinraumes und die Eruptionsgeschichte des Osteifel-Vulkanfeldes. - In: SchIRMER, W. (Hrsg.): Rheingeschichte zwischen Mosel und Maas.- deuqua-Führer 1: 166-190; Hannover 
BrunnaCker, K. (1958): Zur Parallelisierung des Jungpleistozäns in den Periglazialgebieten Bayerns und seiner östlichen Nachbarländer. - Geologisches Jahrbuch, 76: 129-150; Hannover.

- (1990): Gliederung und Dauer des Eiszeitalters im weltweiten Vergleich.- In: H. LIEDKE (Hrsg.): Eiszeitforschung. - Seiten 55-68, Wissenschaftliche Buchgesellschaft Darmstadt.

Connard, N. J., Bosinski, G. \& Adler, D. (1995): Koblenz-Metternich.- In: Schirmer, W. (Hrsg.): Quaternary Field Trips in Central Europe. Vol. 2: 882-885; München.

Dansgaard, W., Johnson, S. J., Clausen, H. B., Dahl-Jensen, D., Gundestrle, N. S., Hammer, C. U., Hvidberg, C. S., Steffensen, J. P., Sveinbjörnsdottir, A. E., White, J., Jouzel, J. \& Bond, G. (1993): Evidence for general instability of past climate from a 250-kyr ice-core record.- Nature, 364: 218-220.

FreChEN, M. (1994): Thermolumineszenz-Datierungen an Lössen des Tönchesberges aus der Osteifel.- Eiszeitalter und Gegenwart, 44: 79-93; Honnover.

- (1995): Eruptionsgeschichte und Deckschichtenfolge der Wannenköpfe-Vulkangruppe in der Osteifel.- Eiszeitalter und Gegenwart, 45: 109-129; Hannover.

- Boenigk, W., Weidenfeller, M. (1995): Chronostratigraphie des „Eiszeitlichen Lößprofils" in KoblenzMetternich.- Mainzer Geowiss. Mitt, 24, S. 155-180, 13 Abb., 2 Tab., Mainz.

— \& Justus, A. (1998): Zur Geologie der Wannen-Vulkangruppe in der Osteifel. - GeoArchaeoRhein, 2: 213-240; Münster.

Hentzsch, B. (1990): Die Lößdeckschichten am Tönchesberg (Osteifel). - In: Schirmer, W. (Hrsg.): Rheingeschichte zwischen Mosel und Maas. deuqua-Führer, 1, 42-46; Hannover.
Johnson, S. J., Clausen, H. B., Dansgaard, W., Gundestrup, N. S., Hammer, C. U., Andersen, U., Andersen, K. K., Hvidberg, C. S., Dahl-Jensen, D., Steffensen J. P., Shoj, H., Sveinbjörnsdottir, A. E., White, J., JouZEL, J. \& Fisher, D. (1997): The 218 record along the Greenland Ice Core Project deep ice core and the problem of possible Eemian climatic instability.Journal of Geophysical Research, 102, N. C12: 26,397-26,410;

Martinson, D. G., Pisias, N. G., Hays, J. D., Imbrie, J., Moore, T.C. \& Shackleton, N. J. (1987): Age dating and the orbital theory of the Ice Ages development of a high resolution 0 to 300,000-year chronostratigraphy. - Quaternary Research, 27: 1-29; Washington.

Reinders, J. \& Hambach, U. (1995): A geomagnetic event recorded in loess deposits of the Tönchesberg (Germany): identification of the Blake magnetic polarity episode. - Geophysical Journal International, 122: 407-418.

Semmel, A. (1968): Studien über den Verlauf jungpleistozäner Formung in Hessen. - Frankfurter geographische Hefte, 45: 1-133; Frankfurt a.M.

ZÖller, L., Conard, N. J. \& Hahn, J. (1991): Thermoluminescence dating of Middle Palaeolithic open airsites in the Middle Thine Valley/Germany.- Naturwisschenaften, 78: 408-410; Berlin; Heidelberg.

Manuskript eingegangen am 10. März 1999 